\title{
Tactical Runway Scheduling for Demand and Delay Management
}

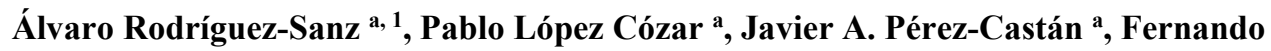 \\ Gómez Comendador a \\ a Universidad Politécnica de Madrid, 28040, Plaza Cardenal Cisneros N3, Madrid, Spain. \\ 1 e--mail: alvaro.rodriguez.sanz@upm.es
}

\begin{abstract}
Airports are limited in terms of capacity. Particularly, runways can only accommodate a certain number of movements (arrivals and departures) while ensuring safety and determined operational requirements. In such a constrained operating environment, any reduction in system capacity results in major delays with significant costs for airlines and passengers. Therefore, the efficient operation of airports is a critical cornerstone for demand and delay management of the whole air transportation system. Runway scheduling deals with the sequencing of arriving and departing aircraft at airports such that a predefined objective is optimized subject to several operational constraints, like the dependency of separation on the leading and trailing aircraft type or the runway occupancy time. Scheduling arrivals and departures at runways is a complex problem that needs to address diverse and often competing considerations among involved flights. In the context of the Airport Collaborative Decision Making (A-CDM) programme, airport operators and air navigation service providers require arrival and departure management tools that improve aircraft flows at airports. Airport runway optimization, as the main element that combines airside and groundside operations, is an ongoing challenge for air traffic management. By considering real airport performance data with scheduled and actual movements, as well as arrival/departure delays, we present a robust model together with an optimization algorithm, which incorporates the knowledge of uncertainty into the tactical operational step. Our model has been validated with real data from a large international European airport in different traffic scenarios. Results are compared to the actual sequencing of flights and show that the algorithm can significantly contribute to the reduction of delay, while adhering as much as possible to the operative procedures and constraints, and to the objectives of the airport stakeholders. Computational experiments performed on the case study illustrate the benefits of this arrival/departure integrated approach.
\end{abstract}

Keywords: airport operations; runway throughput; optimization; arrival management; departure management; A-CDM.

\section{Introduction and problem statement}

Airports are limited in terms of capacity. Particularly, runways can only accommodate a certain number of movements (arrivals and departures) while ensuring safety and determined operational requirements [1]. In such a constrained operating environment, any reduction in system capacity results in major delays with significant costs for airlines and passengers [2-4].

Air transport delays in Europe are a major concern for the industry. Not only is it a painful inconvenience for the actors, but delays also induce large costs for the airlines, their customers and the community as a whole. The total annual cost of air traffic delays to the European economy is around $€ 17.6 \mathrm{bn}$ in 2018 [5]. It is estimated that $70 \%$ of these costs are a result of delays related to ground operations [5]. Capacity depends on infrastructure, operating procedures and operating conditions [6,7]. On-time performance is highly sensitive to airport capacity and flight schedules [8-10]. Runway has been

Content from this work may be used under the terms of the Creative Commons Attribution 3.0 licence. Any further distribution of this work must maintain attribution to the author(s) and the title of the work, journal citation and DOI. 
identified as a main bottleneck in airport operations. Then, increased runway capacity is critical to providing increased overall capacity in the air traffic system [11].

Facing this challenge, demand management can control congestion and match airline scheduling preferences [12]. Runway scheduling techniques belong to a demand management approach of airport management $[12,13]$. Runway scheduling deals with the sequencing of arriving and departing aircraft at airports such that a predefined objective (e.g. delay reduction or throughput maximization) is optimized subject to several operational constraints, like the dependency of separation on the leading and trailing aircraft type or the runway occupancy time $[14,15]$.

Usually, the objective of the scheduling problem is to find a sequence of aircraft operations on one or several runways that minimizes the total makespan, given uncertain aircraft availability at the runway [16]. However, maximizing runway usage needs a trade-off between the runway throughput and the potential delays that may arise $[17,18]$. We focus our objective in minimizing delays given a predefined flight schedule (tactical adjustments). From a set of given flight plans, and considering different operational constraint and uncertainties, we will implement a scheduling algorithm that could be part of the Air Traffic Control (ATC) management tools: we aim for a holistic view that integrates the Arrival Manager (AMAN) with the Departure Manager (DMAN). This model may act as a tactical runway scheduling methodology for reducing delays while managing runway usage. Therefore, our approach to the runway scheduling problem seeks to find a sequence of aircraft operations that ensures an effective runway usage, reduces delays and fulfils the required operational constraints, given uncertain aircraft availability at the runway. However, the planning algorithm needs to be implemented in the real world, where input data (as the estimated time of arrival/departure of an aircraft) face disturbances or changes over time: Our optimization model has been validated with real data from a large international European airport (Alicante-Elche) in different traffic scenarios. In this work, we study the runway scheduling problem at a tactical phase, to consider traffic deviations from the initial planned schedule. By considering real airport performance data with scheduled and actual movements, as well as arrival/departure delays, we present a robust model together with an optimization algorithm, which incorporates the knowledge of uncertainty into the tactical operational step.

\section{Background}

The efficient operation of airports is a critical cornerstone for demand and delay management of the whole air transportation system [13]. Scheduling arrivals and departures at runways is a complex problem that needs to address diverse and often competing considerations among involved flights [14]. In the context of the Airport-Collaborative Decision Making (A-CDM) programme [19], airport operators and air navigation service providers require arrival and departure management tools that improve aircraft flows at airports. Airport runway optimization, as the main element that combines airside and groundside operations, is an ongoing challenge for air traffic management.

Several approaches for the runway scheduling problem have been followed over the years (see [14] for an extensive review of the topic): dynamic programming (a general optimization technique for making sequential decisions), branch-and-bound, branch-and-price, heuristics, metaheuristics (genetic algorithms, ant colony optimization, tabu search, simulated annealing) and queuing theory.

Restrictions for the models and solutions arise from the main characteristic of the problem: the spatial boundary (airport runway with continuous demand feeding), the time boundary (runway scheduling at a tactical phase, to consider traffic deviations from the initial planned schedule), the scope (arrival and departure integrated approach), the airfield layout (availability of runways and taxiways), the operational constraints (separation categories, runway occupancy time) and the timeliness of information (we consider flight plans as an input).

To test the goodness of the proposed models and verify its practical application we will apply the runway scheduling methods to a case study: Alicante-Elche Airport. It is a large international European airport: traffic data in 2019 registered $15,047,840$ passengers $(+7.6 \%$ vs 2018$)$ and 101,408 movements $(+4.8 \%$ vs 2018). The peak traffic month in 2019 was July 2019 with 11,066 movements ( $+5.4 \%$ vs 2018) [20]. An initial traffic characterisation analysis shows that the airport presents high seasonality and high percentage of low-cost traffic (which implies adjusted turnaround times and relevance of delays), with 
$89 \%$ of international passengers (2019) [21]. These factors have a key impact on runway scheduling [14]. Declared capacity (slots in summer 2019 season) was 19 ARR/19DEP/36TOT [20]. Average delays for Alicante are above the operational threshold of $15 \mathrm{~min}$ (18.6 min average delay per departure, 35.6 min average delay per delayed departure, 52.4\% delayed departures and $9.3 \mathrm{~min}$ average reactionary delay per departure) [22]. The airport ranked the $20^{\text {th }}$ position in registered delays in the European Civil Aviation Conference (ECAC) area [22]. Apart from these delay issues, the expected traffic for the airport (forecasted pre-COVID) exceeds declared/practical capacity [23]. Therefore, there is an opportunity to manage runway scheduling to improve the airport's performance.

\section{Objectives}

Main objectives of the study arise from the needs observed in the literature review of the subject. The gaps we aim to fill are:

- Develop a mathematical model for the integrated arrival management (AMAN) and departure management (DMAN) problem (runway scheduling) and a solution algorithm based on a heuristic decomposition of the integrated problem.

- Apply multi-criteria and multi-constraints levels to solve the optimization problem: find a solution that fulfils both operational constrains and targets (maximise runway usage while minimising the cost of delay).

- Provide a management mechanism that protects against tactical uncertainties, reduces delays and provides more stable operations.

- The proposed algorithms can be used to evaluate past operations (post-operational tool) or as a decision-making tool in tactical phase.

\section{Data management and methodology}

\subsection{Data}

The data preparation phase covers all activities required to set up the final dataset from the initial raw aircraft operational runway and final approach radar data.

We generated a dataset of 101,021 flights (50,500 arrivals and 50,521 departures) for Alicante-Elche Airport for the year 2019. This dataset includes real airport performance data with scheduled and actual movements, as well as arrival / departure delays (Table 1). Therefore, we combine delay and capacity metrics.

Delay is the time lapse which occurs when a planned event does not happen at the planned time. It can be positive (actual time later than scheduled time) or negative (scheduled time later than actual time).

Table 1. Explicative variables of the model.

\begin{tabular}{|c|c|}
\hline Type of data & Information \\
\hline Airport infrastructure & $\begin{array}{l}\text { Runway and stand use (terminal area). } \\
\text { Runway declared capacity (arrivals, departures and total). }\end{array}$ \\
\hline Airline & $\begin{array}{c}\text { Operator, type (Low Cost Carrier/Network/Cargo/General Aviation) and associated } \\
\text { handling agent. }\end{array}$ \\
\hline Aircraft & $\begin{array}{l}\text { Model, wake turbulence category (Super Heavy/Heavy/Medium/Light), size (Narrow/Wide } \\
\text { body) and registration number. }\end{array}$ \\
\hline Flight & Flight number, type (commercial or private) and Air Traffic Control (ATC) call sign. \\
\hline Route & Origin and destination, category (Domestic/European/Long Haul). \\
\hline $\begin{array}{l}\text { Operational times \& } \\
\text { regulations }\end{array}$ & $\begin{array}{l}\text { Date, aircraft milestones, timestamps (schedule adherence), duration of processes, holding } \\
\text { patterns, aircraft separation, number of aircraft queuing for the inbound traffic flow and } \\
\text { ATFCM regulations. }\end{array}$ \\
\hline Delay causes & Delay causes according to the codes developed by IATA [24] \\
\hline
\end{tabular}

\subsection{Methodology}

Our approach transforms the planning problem into an assignment problem with side constraints. The coupled landing/take-off problem is solved to optimality by exploiting a time-indexed $(0,1)$ formulation for the problem $[25,26]$, which has been proved successful in scheduling approaches $[27,28]$. Runway scheduling problems can be usefully modelled as dynamic programming problems because the 
algorithms can evaluate current partial solutions independently of the exact sequencing decisions used to form these solutions.

The Binary Integer Linear Programming (BILP) approach allows us to include multi-criteria and multiconstraints levels and, even with some major simplifications, provides fewer sequence changes and target time updates, when compared to the usual approach in which the plan is simply updated in case of infeasibility [27,28]. Thus, the use of robust optimization leads to a protection against tactical uncertainties, reduces delays and provides more stable operations.

The runway scheduling algorithms have been proposed for maximizing runway throughput while minimizing the cost of delays and considering wake vortex separation requirements (operational restrictions). The existing International Civil Aviation Organisation (ICAO) wake vortex separation rules are based solely upon aircraft weight, categorised as Heavy, Medium or Light [29-31]. While safe, in some respects, they are now outdated and lead to over-separation in many instances. This, in turn, diminishes airport capacity and causes unnecessary traffic delays increasing costs, fuel burn and emissions. We apply separation values on the leading and trailing aircraft type based on RECAT-EU European Wake Turbulence Re-categorisation, or Wake RECAT-EU, which provides a safe decrease in separation standards between certain aircraft [32-34].

A statistical analysis of the operational data provides us with information regarding runway use (Table 2), taxiway use (Table 3) and the average Runway Occupancy Time (ROT). Average Arrival ROT (AROT) is $53.59 \mathrm{sg}$. This will represent the main time-constraint (apart from vortex separations) when scheduling operations at the runway.

Table 2. Runway use.

\begin{tabular}{cc}
\hline RUNWAY USE & $(\%)$ \\
\hline RWY 10 & 71.36 \\
RWY 28 & 28.64 \\
\hline
\end{tabular}

Table 3. Taxiway use.

\begin{tabular}{ccccccc}
\hline $\begin{array}{c}\text { EXIT TAXIWAY } \\
\text { USE }\end{array}$ & B (\%) & C (\%) & D (\%) & E (\%) & F (\%) & Total (\%) \\
\hline C2 & 0.00 & 0.03 & 24.57 & 0.50 & 0.45 & 25.55 \\
C4 & 0.29 & 0.59 & 72.50 & 0.19 & 0.03 & 73.59 \\
C5 & 0.01 & 0.00 & 0.56 & 0.00 & 0.00 & 0.57 \\
C7 & 0.00 & 0.00 & 0.28 & 0.00 & 0.00 & 0.29 \\
\hline
\end{tabular}

Figure 1 illustrates the methodological approach: a time-indexed formulation for arrivals and departures time (AMAN/DMAN integrated model).

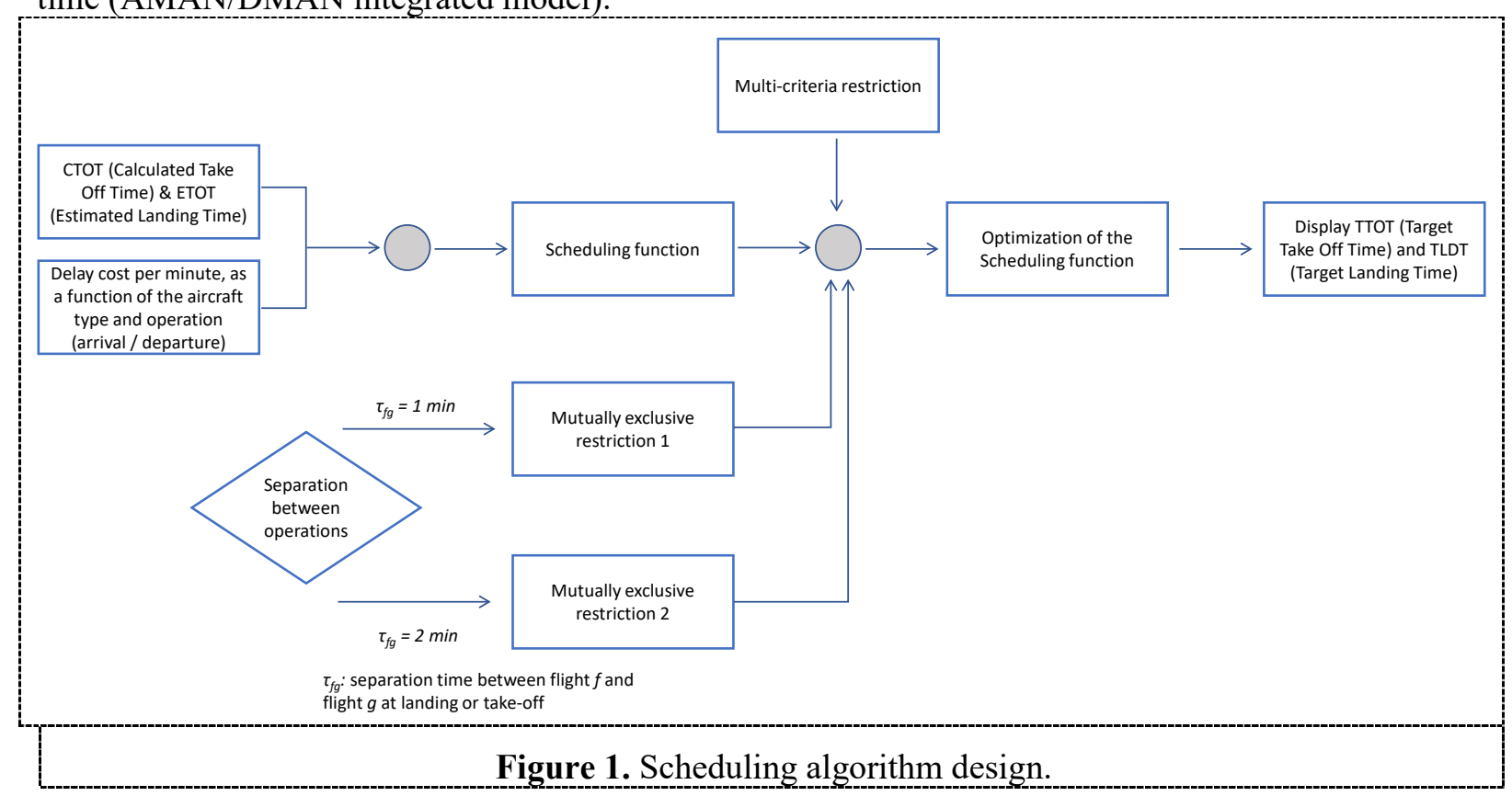


We present here a 0,1 Linear Program to tackle the optimization problem [25-27]. We limit the description to the case of single arrival runway and single departure runway (the two runways coincide for the case study at Alicante). However, the extension to multiple runways is straightforward.

First, we discretize the time horizon and let $H=\{1,2, \ldots\}$ be the periods. We let $F=L U D$ be the set of flights to be controlled in the time horizon $H$, with $L$ be the set of arrival (landing) flights and $D$ be the set of departure flights $(L \cap D=\varnothing)$, hereafter denoted as arrivals and departures.

For each arrival $l \in L, \lambda l$ denotes the target landing time, with $\lambda l \in[\alpha l, \beta l]$, the arrival window associated with $l$; also we let $\omega l$ be the wanted in-block time, i.e. the time the arrival is expected to reach the stand. For each departure $d \in D$, we let $\delta d$ be the target departure time with $\delta d \in[\alpha d, \beta d]$ as the departure window for $d \in D$. Finally, we let $\omega d$ be the target off-block time, namely the time in which departure $d$ will be ready to leave the gate. In our model, a take-off must happen during the departure window.

Finally, when $f$ precedes $g, \tau_{f g}$ denotes the separation time between flight $f$ and flight $g$ at landing or takeoff, depending whether $f$ is arrival or departure, and $g$ is arrival or departure.

For each departure (arrival) $f \in F$ and each time period in $t \in H f$ we introduce a binary variable $x_{f t}$ which is 1 if and only if $f$ takes off (lands) at time $t$. Taking off or landing at time $t$ has a cost $c_{f t}$. For departure $d$ (arrival $l$ ) such cost increases with $|t-\delta d|(|t-\lambda l|)$. For each departure $d$ we introduce a binary variable $y d$ which is 1 if and only if $d$ is dropped. Dropping a departure $d \in D$ has (typically large) cost $w d$.

Then, the constraints can be written as follows:

Each arrival must be assigned a landing time: $\sum_{t \in H_{l}} x_{l t}=1 ; l \in L$ (1)

Each departure must be assigned a departure time or is dropped: $\sum_{t \in H_{d}} x_{d t}+y_{d}=1 ; d \in D$ (2)

A departure $d$ cannot leave the stand before its off-block time $\omega d$, so it cannot take-off before $T X d+$ $\omega d: x_{d t}=0 ; d \in D ; t \in H_{d} ; t<T X_{d}+w_{d}(3)$

Constraints on runway separation between flights become: $x_{f t}+\sum_{k \in\{t, \ldots, t+\tau f g\}} x_{g k} \leq 1 ; t \in$ $H_{f} ;(f, g) \in F x F ; f \neq g(4)$

Finally, the objective is to minimize the cost of dropped flights plus the overall deviation from the wanted arrival and departure times: $\sum_{d \in D} w_{d} y_{d}+\sum_{f \in F, t \in H_{f}} c_{f t} x_{f t}(5)$

For the optimization process, the cost of delay must be considered. 'Schedule delay' is a term in transport modelling which refers to a difference between a desired time of arrival or departure and the actual time. Despite the use of 'delay', it can refer to a difference in either the early or late direction [35]. Hence, they can be positive or negative. Delays can be split between primary and reactionary delays [36-40]: reactionary delays are delays that are caused by the late arrival of aircraft, crew, passengers or loads from a previous journey. Primary delays are all other delays and occur during the turnaround process of the aircraft. The costs of delays can be calculated separately for strategic delays (those anticipated in advance) and tactical delays (those incurred on the day and not scheduled into passenger or airline schedules).

The proposed model considers the cost of delay per type of aircraft, per operation (landing or take off) and per delay category (positive or negative) [36].

For example, at-gate delay for a Boeing B737 (cost per minute) is computed as $c_{l-B 737}=|t-\lambda| *$ $52=[$ Euros $(€)]$.

Please note that we have introduced a binary variable $x_{f t}$ which is 1 if and only if $f$ takes off (lands) at time $t$. Taking off or landing at time $t$ has a cost $c_{f t}$. For departure $d$ (arrival $l$ ) such cost increases with $|t-\delta d|(|t-\lambda l|)$.

\section{Results}

The runway scheduling model was applied to flight plans in several traffic scenarios: peak, average and low-traffic demand periods. Moreover, we performed two different simulations in each scenario, depending on the separation considered between aircraft: simulation $1\left(\tau_{f g}=1 \mathrm{~min}\right.$, as an optimistic scheme) and simulation 2 ( $\tau_{f g}=2 \mathrm{~min}$, as a conservative scheme).

For illustrating the applicability of the model, we present the results for the peak period in traffic, which corresponds to Tuesday 02/07/2019 between $7 \mathrm{AM}$ and $10 \mathrm{AM}$ local time. It is important to also monitor the previous and subsequent hours, as delays can increase after peak periods due to the network effect. 
An analysis of delay records shows that take offs are generating a higher amount of 'positive' delays (219 $\mathrm{min}$ in total) than landings (101 $\mathrm{min}$ in total), which is partly due to a problem in runway scheduling management. 'Positive' flight delays are those that arise from a difference in late direction, they cause significant problems for stakeholders involved; e.g., they affect the operational and financial performance of airports and airlines, schedule adherence and use of resources, passenger experience and satisfaction, and system reliability $[9,41]$.

The algorithm output for a separation between aircraft of 1 min (optimistic scenario) achieves $74 \%$ less delays than the actual operated situation. The model output for a separation between aircraft of 2 min (conservative scenario) achieves 51\% less delays than the actual operated situation.

Apart from using the raw information of delay in minutes as a metric for testing the goodness of the model, we also considered the actual costs of delay (weighting delays by type of aircraft and operation). If we look at the improvement in the cost of delays when following the schedule proposed by the arrival/departure integrated algorithm we get the values included in Figure 2. For the most congested hour (9 AM to 10 AM local time), the scheduling algorithm may allow a decrease of nearly $12,000 €$ in terms of delay costs.

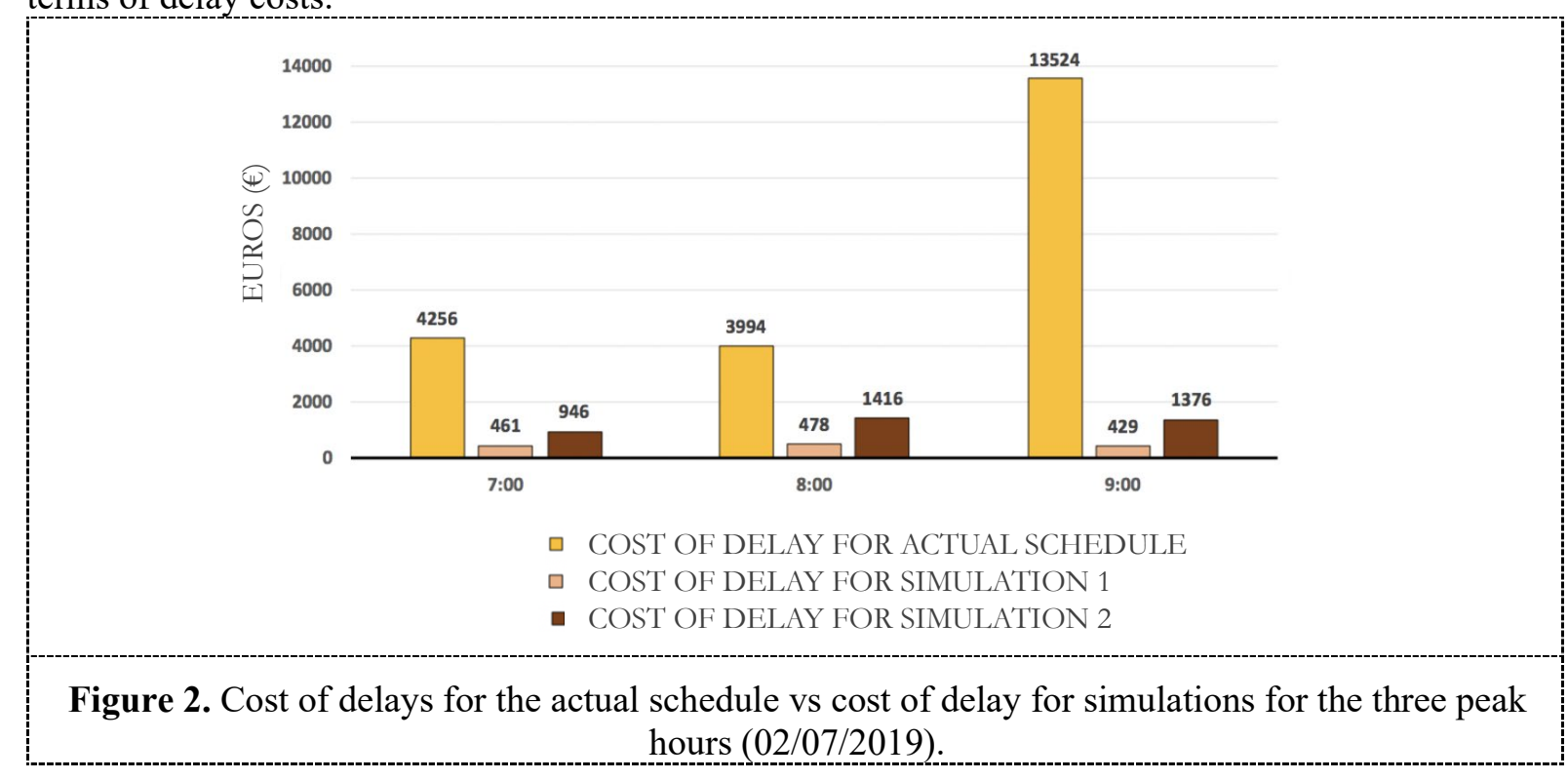

\section{Conclusions and future work}

Runway scheduling techniques belong to a demand management approach of airport management. It can control congestion and match airline scheduling preferences. The arrival and departure scheduling problems are tightly connected (highly dependent on each other for a single runway layout) and should be solved as one. It is common practice of the airports to handle them separately, which prevents the needed global perspective to ensure properly coordinated decisions are made. Our mathematical model integrates runway scheduling for arrival and departures. Particularly, we propose an algorithm to maximise runway usage and minimise delays in a tactical phase.

Our optimization model has been validated with real data from a large international European airport (Alicante-Elche) in different traffic scenarios, where it showed remarkable improvements in punctuality compared to actual sequencing.

Results are compared to the actual sequencing of flights and show that the algorithm can significantly contribute to the reduction of delay, while adhering as much as possible to the operative procedures and constraints, and to the objectives of the airport stakeholders. Regarding punctuality, the proposed algorithm provides a reduction of $50 \%$ of delays on average and a reduction of the makespan by $5-7 \%$. The proposed models ensure a reduction in cost of delays: the impact of delays on passengers and airlines can be significantly reduced (quantified by an average decrease of $60 \%$ in delay costs). 
Our algorithm does not follow gapping / packing sequence patterns (predefined solutions) that may reduce airport performance, the coordination of arrivals and departures provides a global perspective of runway operations and enhances decision-making.

Computational experiments performed on the case study illustrate the benefits of the arrival/departure integrated approach: the proposed algorithm significantly reduces weighted aircraft delay and computes efficient runway schedule solutions within a few seconds and with little computational effort. Therefore, it can be adopted as a decision-making tool in the tactical stage

Future works seeks to:

- Improve the accuracy of the model (more complete testing data and methodological enhancements) and compare the results when the methodology is applied to other airports (generalisation of the case study).

- Optimize runway scheduling by also considering the number of passengers per flight (delay weighting).

- Develop a feedback control loop and analyse potential response strategies / measures (how specific actions impact runway scheduling and capacity / demand management).

- Extend airport characterisation to surface movement (SMAN) and conflict resolution: decompose the problem of routing, sequencing, and conflict resolution in subsequent stages. Possible types of integration include integrating runway scheduling, ground movement control, and gate assignment.

- Analyse the impact of irregular operations and other sources of uncertainty (stochastic approach of the problem), therefore accounting for more operational constraints. In a dynamic environment, uncertainty can be caused by weather (such as wind or snow), the precision of equipment, as well as the variability in pushback times and taxi times for departing aircraft.

- Include more general cost functions: multi-criteria optimisation. Select more objectives that can satisfy the interests of all parties, or provide an acceptable compromise solution.

\section{References}

[1] Horonjeff R M, McKelvey F X, Sproule W J and Young S 2010 Planning and Design of Airports (New York: McGraw-Hill)

[2] Fleurquin P, Ramasco J J and Eguiluz V M 2013 Systemic delay propagation in the US airport network Sci. Rep. 3

[3] Rebollo J J and Balakrishnan H 2014 Characterization and prediction of air traffic delays Transp. Res. Part C Emerg. Technol.

[4] Rodríguez-Sanz Á, Comendador F G, Valdés R A and Pérez-Castán J A 2018 Characterization and prediction of the airport operational saturation J. Air Transp. Manag. 69 147-72

[5] Performance Review Commission 2019 Performance Review Report-An Assessment of Air Traffic Management in Europe during the Calendar Year 2018 (Brussels: European Organisation for the Safety of Air Navigation (EUROCONTROL))

[6] Wells A T and Young S B 2014 Airport Planning \& Management

[7] Ashford N J, Stanton H P M, Moore C A, Coutu P and Beasley J R 2013 Airport Operations (New York: McGraw-Hill)

[8] Wu C L and Caves R E 2004 Modelling and optimization of aircraft turnaround time at an airport Transp. Plan. Technol. 27 47-66

[9] Wu C L 2012 Airline operations and delay management: Insights from airline economics, networks and strategic schedule planning

[10] Bazargan M 2010 Airline Operations and Scheduling (Aldershot: Ashgate)

[11] Balakrishnan H and Chandran B G 2010 Algorithms for Scheduling Runway Operations Under Constrained Position Shifting Oper. Res. 58 1650-65

[12] Gillen D, Jacquillat A and Odoni A R 2016 Airport demand management: The operations research and economics perspectives and potential synergies Transp. Res. Part A Policy Pract. 94 495-513

[13] Jacquillat A and Odoni A R 2017 A roadmap toward airport demand and capacity 
management Transp. Res. Part A Policy Pract.

[14] Bennell J A, Mesgarpour M and Potts C N 2013 Airport runway scheduling Ann. Oper. Res. 204 249-70

[15] Bennell J A, Mesgarpour M and Potts C N 2011 Airport runway scheduling 4OR-Q J Oper $\operatorname{Res} 9$ 115-38

[16] Heidt A, Helmke H, Kapolke M, Liers F and Martin A 2016 Robust runway scheduling under uncertain conditions J. Air Transp. Manag. 56 28-37

[17] Solak S, Solveling G, Clarke J P B and Johnson E L 2018 Stochastic runway scheduling Transp. Sci. 52

[18] Solveling G, Solak S, Clarke J P and Johnson E 2011 Runway operations optimization in the presence of uncertainties J. Guid. Control. Dyn. 34 1373-82

[19] Eurocontrol 2006 Airport CDM Implementation Eurocontrol, Brussels, Belgium

[20] AENA 2020 Air Traffic Statistical Information

[21] AENA 2020 Airport information $h t t p: / / w w w . a e n a . e s / e n / a i r l i n e s / a i r p o r t s-n e t w o r k . h t m l$

[22] EUROCONTROL 2020 CODA (Central Office for Delay Analysis) Digest: All-Causes Delay and Cancellations to Air Transport in Europe - 2019 (Brussels: European Organisation for the Safety of Air Navigation)

[23] AENA 2020 Airport information http://www.aena.es/en/airlines/airports-network.html

[24] IATA 2015 Airport Handling Manual 35th edition (Montreal: International Air Transport Association)

[25] Nemhauser G and Wolsey L 1988 Integer and Combinatorial Optimization

[26] Bertsimas D and Tsitsiklis J N 1997 Introduction to Linear Optimization (Massachussets: Athena scientific)

[27] Kjenstad D, Mannino C, Nordlander T E, Schittekat P and Smedsrud M 2013 Optimizing AMAN-SMAN-DMAN at Hamburg and Arlanda airport SIDs 2013 - Proceedings of the SESAR Innovation Days

[28] Bertsimas D, Lulli G and Odoni A 2011 An Integer Optimization Approach to Large-Scale Air Traffic Flow Management Oper. Res. 59 211-27

[29] ICAO 1998 Doc 9689-AN/953 - Manual on airspace planning methodology for the determination of separation minima

[30] ICAO 2016 Doc. 4444: Procedures for Air Navigation Services - Air Traffic Management (Montreal: International Aviation Civil Organization)

[31] ICAO 2006 Document 8168: Procedures for Air Navigation Services - Aircraft Operations 5th edition (Montreal: International Civil Aviation Organization)

[32] SESAR 2014 SESAR Concept of Operations Step 2 Edition 2014 (Ed. 01.01.00) (Brussels: SESAR Joint Undertaking)

[33] SESAR Joint Undertaking 2017 SESAR 2020 Concept Of Operations

[34] SESAR Joint Undertaking 2019 SESAR Solutions Catalogue 2019. 3rd Ed.

[35] Small K A 1982 The scheduling of consumer activities: work trips. Am. Econ. Rev. 72 467-79

[36] Cook A and Tanner G 2014 European airline delay cost reference (Brussels: University of Westminster comissioned by EUROCONTROL Performance Review Unit)

[37] Cook A, Tanner G and Enaud P 2010 Quantifying Airline Delay Costs - The Balance Between Strategic and Tactical Costs Proceedings of the 14th Air Transport Research Society (ATRS) World Conference (Porto, Portugal)

[38] Cook A, Tanner G and Lawes A 2012 The hidden cost of airline unpunctuality J. Transp. Econ. Policy 46 157-73

[39] Jetzki M 2009 The propagation of air transport delays in Europe (Thesis in the Department of Airport and Air Transportation Research. RWTH Aachen University)

[40] Institut du Transport Aérien (ITA) 2000 Costs of Air Transport Delay in Europe. Final report (Brussels: European Organisation for the Safety of Air Navigation (EUROCONTROL))

[41] Belobaba P, Odoni A and Barnhart C 2015 The Global Airline Industry ed Wiley (New York) 